\title{
Crecimiento de startups de agrobiotecnología: una aproximación a partir de un estudio de casos múltiple
}

Growth of agrobiotechnology startups: an approximation from a study multiple cases

\author{
Elsie Veronica Muñoz Arellano \\ elsie.munoza@pucp.pe \\ Thania Felices Ochoa \\ thania.felices@pucp.pe
}


Las agrobiotech startups son un modelo de negocio disruptivo que brinda alternativas mediante productos o servicios a diferentes tipos de cultivo en términos de productividad, calidad y/o sostenibilidad. El desarrollo de la agrobiotecnología podría contribuir a solucionar los desafíos que presenta el sector agrario peruano, contrarrestando de forma efectiva la pobreza y el hambre en el país. Este articulo aporta al conocimiento de las agrobiotech startups, pues identifica y describe los elementos clave de crecimiento para este tipo de startups en el ecosistema emprendedor peruano. Para ello, se realizó un estudio de casos múltiple con tres agrobiotech startups ganadoras del concurso Startup Perú o Reto Bio. Los hallazgos demuestran que, por la particularidad del sector, la geografía y la tecnología aplicada, se identifican elementos referentes al entorno, negocio y al propio emprendedor, con participación y apreciación particular.

Palabras clave: agrobiotecnología, startups , crecimiento, ecosistema emprendedor

The agrobiotech startups are a disruptive business model that provides alternatives through products or services to different types of crops in terms of productivity, quality and/or sustainability. The development of agrobiotech could contribute to solve challenges that the Peruvian agricultural sector faces, counteracting poverty and hunger effectively. This article contributes to the knowledge of agrobiotech startups, since it identifies and describes the key growth elements for this type of startups in the Peruvian entrepreneurial ecosystem. For this, a multiple case study was carried out with three agrobiotech startups winners of the Startup Peru or Reto Bio contest. The findings show that, due to the particularity of the sector, the geography and the applied technology, elements related to the environment, business and the entrepreneur itself are presented, with particular participation and appreciation. 


\section{Introducción}

En el ecosistema peruano emprendedor se ha identificado un nuevo actor: las startups de agrobiotecnología — también llamadas agrobiotech startups—, las cuales utilizan la biotecnología para ofrecer productos y servicios innovadores en el sector agrícola, orientados a la sostenibilidad de los cultivos y, consecuentemente de la biodiversidad (CONAM, 1997; Pastor, 2005). Si bien la representatividad de este tipo de startups es baja en el país, estas han logrado captar el interés de entidades públicas y privadas por el impacto y necesidad de los productos y servicios que han desarrollado (Startup Perú, 2019). Asimismo, en ciertos países de Europa y Asia se ha explorado a alto nivel el crecimiento y expansión de la biotecnología, lo cual ha generado oportunidades de investigación para su desarrollo (Dorockis \& Bogúa, 2014).

El objetivo de este trabajo es identificar y describir los elementos clave de crecimiento mediante un estudio de casos múltiple con tres startups de agrobiotecnología ganadoras del concurso StartUp Perú o Reto Bio. Para ello, se toma como base el modelo de Vier (2016), en el cual se considera que hay elementos que deben tenerse presentes en la gestión y el fomento de este tipo de startups por el impacto que podrían tener para su escalabilidad en el mercado. Además, se asume que las startups de agrobiotecnología ganadoras de StartUp Perú o Reto Bio son conscientes de la importancia de conocer su entorno, su organización y sus propias propuestas como emprendedoras; y, por ende, se encuentran analizando constantemente estos elementos en diferentes niveles, concluyendo en priorizaciones y estrategias.

Por otro lado, este estudio es relevante por tres razones. En primer lugar, dado que la biotecnología se considera la fuente de soluciones a los desafíos que el sector agrario presenta y que, gracias a determinados agentes de cambio, ha iniciado una revolución mundial (Newell, 2007). En ese sentido, se considera que el avance de esta rama de la ciencia puede ayudar a los países en desarrollo a vencer la pobreza y el hambre de forma más efectiva (Chaturvedi, 2004, citado en Dorockis \& Bogús, 2014).

En segundo lugar, en el Perú se han identificado límites para el desarrollo de la biotecnología y la innovación que deriva de ella (Concytec, 2016), lo cual puede impactar en el crecimiento de este tipo de startups. Por lo mismo, es necesario que se identifiquen los principales elementos que se presentan en su proceso de crecimiento para clasificarlos y evaluarlos, ya que esto facilitará la toma de decisiones estratégicas, la formulación de ventajas competitivas y la gestión (Teece, 2018). También se identificó que los elementos de crecimiento no han sido estudiados a profundidad en startups de agrobiotecnología, a pesar de que existen estudios relacionados al tema para diferentes regiones y tipos de empresas (Gertler \& Levitte, 2006). Al respecto, un reciente estudio peruano que trata sobre este tema en tech startups concluye que se requiere una comprensión más profunda para este tipo particular de startups en lo que concierne a sus elementos clave de crecimiento (Hernández \& González, 2016).

Finalmente, en esta investigación primero se presenta el marco teórico sobre crecimiento y los elementos clave para cada etapa; luego, se describe el estudio empírico realizado y los resultados obtenidos; y, después, estos se interpretan y se discuten los hallazgos. Por último, se mencionan las conclusiones y limitaciones del estudio. 


\section{Marco teórico}

\subsection{Crecimiento y agrobiotecnología}

El crecimiento de una organización es un fenómeno de las ciencias de la gestión que diversos académicos han estudiado desde diferentes enfoques y perspectivas intentando entender y definir su naturaleza (Canals, 2002; Santana et al., 2006; Capelleras \& Kantis, 2009; Davidsson et al., 2010; Mckelvie \&Wiklund, 2013; Seclen, 2016a). Alineado con ello, se rescatan estudios que se han enfocado en evaluar el crecimiento e impacto de las empresas de agrobiotecnología a alto nivel (Dorockis \& Bogús, 2014; González, 2011) y, en algunos casos, centrados específicamente en el caso de las startups desde diferentes enfoques y contextos (Newell, 2007; Rank, 2014; Honjo \& Nagaoka, 2018).

En las fuentes consultadas se identificaron dos enfoques: uno que define al crecimiento como resultado (Santana et al., 2006) y otro como un proceso (Canals, 2002; Santana et al., 2006). En el presente artículo se considera el segundo enfoque, pues el primero se rige por un análisis lineal y unidimensional que, al no considerar hitos en el crecimiento, en algunos casos imposibilita su medición y posterior comparación (Wiklund et al., 2013; Mckelvie \& Wiklund, 2013). Por lo tanto, se considera que el crecimiento es un proceso iterativo y dinámico que se divide en etapas temporales no lineales (Vier, 2016). Asimismo, de las diferentes formas de estructurar estas etapas, la más cercana al sujeto de estudio es la de Hernández y González (2016) debido a que su investigación se extiende al grupo tech startups, al que pertenecen las agrobiotech startups. Estos autores establecen cuatro etapas: definición, validación, eficiencia y crecimiento.

Centrándonos en la etapa de crecimiento, Hernández y González (2016) indican que cuando la startup llega a este punto, enfrenta la oportunidad de expandir el negocio a otros mercados y presenta la viabilidad suficiente para adquirir el capital necesario para concretarlo. En la presente investigación, se enriquece el análisis de la etapa de crecimiento que presentan las startups con el modelo de Scott y Bruce (1987), quienes toman en consideración determinadas variables —alineadas a Hernández y González (2016)— que permiten un análisis holístico de las startups.

Se entiende como startup a una organización nueva y temporal que tiene como base la innovación y tecnología que se reflejan en iniciativas de alta recompensa, pero, a la par, de alto riesgo (Innóvate Perú, 2019; Blank \& Dorf, 2012). En los últimos años, estos actores han ingresado al sector agrícola peruano y han logrado establecerse en determinados nichos de mercado como startups de agrobiotecnología. En el Perú, este sector es uno de los más relevantes para la economía peruana debido a la riqueza de recursos que presenta, los cuales se busca explotar de forma sostenible y competitiva (CEPAL et al., 2019) desde cuatro pilares importantes, entre los cuales se encuentran el financiamiento y la tecnología (Minagri, 2019).

La biotecnología es un segmento perteneciente a este último pilar, el tecnológico, que busca cumplir los objetivos planteados líneas arriba. En la presente investigación, la biotecnología se entiende como toda aplicación que combina las ciencias de la vida y la tecnología que permite modificar organismos o microorganismos para obtener uno nuevo, mejorar las características del propio mediante diferentes técnicas, o generar otros 
recursos o instrumentos que tienen aplicación o relación directa con ellos (Aguinaga, 1988; CONAM, 1997; Romero, 2008). Las startups de agrobiotecnología se diferencian por la aplicación de este tipo de tecnología y la innovación de productos que se obtiene. Además, se rescata el que estas startups presenten en su entorno (Concytec, 2016), en su propia organización (Gertler \& Levitte, 2006; Dorockis \& Bogus, 2014) y en el perfil de sus emprendedores (Zucker et al., 1998; Staniewski, 2016; Dorockis \& Bogus, 2014) elementos particulares que participan en su proceso de crecimiento.

Ante ello, Hernández \& González (2016) sugieren realizar un estudio en el Perú debido a la creciente presencia de la biotecnología en el mercado. De acuerdo a Vier (2016), estos elementos se han clasificado en tres grupos que permiten el análisis multidimensional que este fenómeno requiere. Estos grupos son el emprendedor, el negocio y el entorno, dentro de los cuales se presentan determinados elementos que han sido enriquecidos con la revisión de la literatura sobre este tipo de tecnología y sobre startups para rescatar sus principales características y formas de participación. A continuación, se presentan detalladamente estos elementos en los respectivos grupos.

\subsection{Elementos clave de crecimiento}

Se rescatan determinados estudios que no necesariamente precisaron el mismo sujeto de estudio o que se enfocaron en determinados elementos; sin embargo, estos enriquecen el marco teórico de Vier (2016), el cual es la base de análisis de este estudio. A continuación, se presentan los elementos clave de crecimiento por cada grupo considerados para la presente investigación.

\subsubsection{Grupo emprendedor}

Uno de los elementos identificados es el nivel educativo que presentan los emprendedores (E1) (Hidalgo et al., 2014; Vier, 2016), el cual es especializado para el tipo de tecnología que se implementa y los recursos que se gestionan (Zucker et al., 1998; Staniewski, 2016; Dorockis \& Bogus, 2014). Este elemento es necesario por la base de conocimientos que se requiere para tener un desarrollo sostenible y gestionar positivamente el impacto de la biotecnología (Pastor, 2005).

Adicionalmente a ello, se requiere como complemento una formación -o conocimientos - en negocios (E2) e inversiones (E3) debido a que se presentan funciones y roles direccionados a gestionar el emprendimiento o, en este caso, la startup (Costa et al., 2004). Como parte de todo ello, se tienen determinadas variables que sostienen el relacionamiento del emprendedor con otros actores estratégicos, como la red de contactos del emprendedor, sus habilidades blandas y analíticas, y la capacidad de captar financiamiento (Baptista et al., 2014; Vier, 2016). De las habilidades blandas a desarrollar, una de las que determina la diferenciación de la startup es el liderazgo (E4), ya que representa directamente al capital humano, brinda la visión que se quiere tener en la startup y es el primer frente en la toma de decisiones (Zucker et al., 1998).

Como complemento, se presenta la experiencia y trayectoria de los emprendedores (E5), las cuales brindan información de los conocimientos y habilidades que han adquirido 
(Baptista et al., 2014; Oe \& Mitsuhashi, 2013; Hernández \& González, 2016; Vier, 216). Ambos elementos son importantes y se deben tomar en consideración por los riesgos sociales, económicos y ambientales que implica el desarrollo de productos aplicando la biotecnología en este sector (Staniewski, 2016).

Por último, se presenta la aspiración profesional y personal del emprendedor (E6) (Vier, 2016). En el caso de la primera, se identificó una relación con la actitud que presenta el emprendedor ante el riesgo, la cual conlleva a la startup a tener una variedad de resultados (Tan et al., 2007). La segunda, por su parte, abarca la motivación intrínseca de los emprendedores; es decir, los logros personales a obtener, dentro de los cuales se incluyen sus motivaciones para iniciar el negocio (Tan et al., 2007; Vier, 2016).

En resumen, se identificaron seis elementos clave de crecimiento en el grupo del emprendedor para este tipo de startups y que enmarcan no solo el nivel técnico, sino también habilidades blandas. En el siguiente punto, se complementa esta información con los elementos identificados en el grupo del negocio.

\subsubsection{Grupo de negocio}

En este grupo se contempla a la par los elementos de nivel educativo y la experiencia de los colaboradores y miembros de la organización (N1), los cuales deben estar alineados a lo que requiere la organización en términos de especialización y rango de experiencia, según las funciones y los roles a asumir (Korunka et al., 2010; Vier, 2016). Por fines prácticos, se considera al aprendizaje y la experiencia como un solo elemento para este grupo.

Además, se identificó el elemento de conocimiento individual o único (N2), que representa aquellos conocimientos que el colaborador o equipo de colaboradores (Becker, 1975, citado en Colombo \& Grilli, 2005) han desarrollado y que se implementan principalmente para la innovación de productos (Ryan \& Phillips, 2004; Staniewski, 2016; Vier, 2016). De acuerdo a cómo se gestione dicho conocimiento, se puede desarrollar una ventaja competitiva y se pueden potenciar las capacidades del equipo (Subramaniam \& Youndt, 2005; Teece, 2018). Frente a ello, se identificó otro elemento: la estrategia del capital humano (N3) (Coleman, 2000; Vier, 2016).

Debido a la heterogeneidad que el grupo humano presenta, un factor necesario en una startup por la diversidad de roles y funciones existentes, se requiere evaluar estrategias para retener y potenciar el valor que los colaboradores generan, particularmente en este tipo de industrias de conocimiento intensivo (Kaiser \& Muller, 2015). El impacto de ello se da en diferentes formas, entre las cuales destaca la construcción de redes estratégicas (Davidsson \& Honig, 2003; Vier, 2016)

Para el direccionamiento de esta ventaja, se debe considerar la demanda de la línea de negocio en donde se ha establecido la startup; en otras palabras, su formato o línea de negocio (N4). Este cuarto elemento abarca el modelo de negocio de la startup y el sector al que ha ingresado con sus respectivos riesgos. Asimismo, buscando complementar este análisis, se identificó el siguiente elemento: la gestión de los recursos financieros (N5). Para que esta sea viable, se debe mantener y aplicar en la búsqueda de recursos activos y pasivos hasta llegar al punto de equilibrio y dar soporte al logro y planteamiento de objetivos (Vier, 2016). 
Por otro lado, se identificó otro elemento relacionado a los procesos centrales de una organización: la relación con los proveedores (N6) (Vier, 2016). Este elemento cumple un rol estratégico en las actividades de la empresa debido a su impacto en la calidad del servicio y/o los productos que ofrece la organización; es decir, en el valor final y percibido por el cliente (Vier, 2016; Beekman \& Robinson, 2004). Asimismo, se rescata el último elemento, el cual es la gestión de la innovación (N7). Este elemento abarca desde la investigación hasta la comercialización de un producto en el mercado (Seclen \& Barrutia, 2019). En la biotecnología, a pesar de que la investigación e información recopilada llega a escala global gracias a los medios desarrollados, cada país tiene determinadas preferencias en la interacción con otros actores, lo cual se superpone a la cercanía geográfica para facilitar el proceso de innovación (Gertler \& Levitte, 2006).

En resumen, se identificaron siete elementos clave de crecimiento en el presente grupo, siguiendo a Vier (2016), y las características propias del negocio según estudios centrados en biotecnología y/o startups. En el siguiente punto, se presentan los elementos identificados en el entorno.

\subsubsection{Grupo del entorno}

Uno de los elementos identificados es el clúster (T1), dado que es un referente de la situación del sector en el que se encuentra la empresa (Vier, 2016). En el Perú no se tiene identificado un clúster en este sector, pero sí se tienen nichos de mercado en los cuales hay potencial para desarrollarse (Moodysson et al., 2006). Otros dos elementos identificados, que cumplen un rol de facilitadores o mentores, son los inversionistas ángeles y el venture capital (T2), actores que dan soporte mediante asesoría y/o capital financiero al crecimiento de una startup (OCDE, 2016; Vier, 2016).

De igual manera, se identificaron elementos cuyo objetivo es expandir la influencia de estos modelos de negocio mediante transferencia tecnológica y educación en la cultura emprendedora (Bandera \& Thomas, 2018), entre los cuales destacan las universidades y los centros de investigación (T3) (Hernández \& González, 2017; Vier, 2016). El primer elemento, las universidades, hace referencia a actores primarios del desarrollo de este tipo de cultura y proveedores de la infraestructura que facilita la investigación e innovación (Dorockis \& Bogus, 2014), lo cual conlleva un rol como proveedores de fuerza laboral y especializada, incluyendo la que requieren las startups de agrobiotecnología (Gertler \& Levitte, 2006). Por otro lado, un centro de investigación es referente por el flujo de información que comparte y genera, lo cual facilita la búsqueda y sustento de nuevos desarrollos a aquellos enfocados en la innovación en el sector agrícola y biotecnología (Tostes, 2014). En el Perú, esto se ha reforzado mediante nuevos proyectos y programas para los agricultores y emprendedores del sector, en conjunto con entidades públicas y privadas a nivel nacional e internacional (Tostes, 2014; INIA, 2018; Minagri, 2019).

De la participación activa del sector público y las entidades financieras se rescatan dos elementos: por un lado, los incentivos fiscales y la tributación fiscal (T4); y, por otro, las políticas y normas públicas a nivel nacional e internacional (T5). El primer elemento enfatiza los beneficios fiscales que los emprendimientos en este sector reciben, mientras que el segundo se enmarca en las otras políticas implementadas (Vier, 2016), como la industrial, 
la sanitaria y la de consumo, cuyo objetivo es proteger al consumidor, los agricultores y los recursos naturales mediante la promulgación de marcos de servicio a seguir.

Para facilitar el cumplimiento de estas reglas, se identificó otro elemento: la disponibilidad de recursos (T6). En el caso de este tipo de startups, los recursos principalmente demandados son determinados materiales o insumos, equipos sofisticados y ambientes de trabajo (Hidalgo et al., 2014; Vier, 2016; Tripathi et al., 2018). Asimismo, este elemento depende del dinamismo del sector y de la situación del mercado (T7) en el que se encuentra el negocio, e incluye la oferta de capital humano especializado en estos temas (Gertler \& Levitte, 2006; Vier, 2016).

Por último, se identificó un elemento que representa la aceptación de los productos que ofrece la startup en el mercado: la relación entre oferta-demanda (T8) (Menrad, 2000; Hidalgo et al., 2014). Entre el anterior y este elemento, este último prevalece debido a que la aceptación del producto y su demanda garantizan la continuación del negocio al generar flujo de caja; es decir, sustento económico para sus operaciones y escalabilidad (Vier, 2016; Menrad, 2000; Trigo \& Villareal, 2009; Woolfson, 2013; Portafolio, 2018).

En resumen, en este grupo se han identificado ocho elementos clave de crecimiento para las startups de agrobiotecnología. Igualmente, sumando los tres grupos, se han identificado 21 elementos (ver tabla 1). Para estos puntos, se ha tomado en consideración que el crecimiento es un proceso iterativo y dinámico (OCDE, 2016). Por ello, para el análisis respectivo, se considera a startups de agrobiotecnología en etapa de crecimiento y que están activas actualmente en Perú.

\section{Tabla 1. Elementos clave de crecimiento identificados en la literatura}

\begin{tabular}{|c|c|c|}
\hline Grupo & Elementos & Código \\
\hline \multirow[t]{6}{*}{ Emprendedor } & Nivel educativo y de especialización & E1 \\
\hline & Conocimientos en negocios y gestión de empresas & E2 \\
\hline & Conocimientos en inversiones & E3 \\
\hline & Liderazgo & E4 \\
\hline & Experiencia y trayectoria & E5 \\
\hline & Aspiración profesional y personal & E6 \\
\hline \multirow[t]{7}{*}{ Negocio } & Aprendizaje y experiencia & N1 \\
\hline & Conocimiento individual & N2 \\
\hline & Estrategia del capital humano & N3 \\
\hline & Naturaleza o formato del negocio & N4 \\
\hline & Estrategias de financiamiento para inversión y expansión & N5 \\
\hline & Relación con proveedores & N6 \\
\hline & Gestión de la investigación, innovación y desarrollo & N7 \\
\hline
\end{tabular}




\begin{tabular}{l|l|c} 
Entorno & Clústeres & T1 \\
\cline { 2 - 3 } & Inversionistas ángeles y venture capital & T2 \\
\cline { 2 - 3 } & Universidades y centros de investigación & T4 \\
\cline { 2 - 3 } & Incentivos fiscales y tributación fiscal & T5 \\
\cline { 2 - 3 } & Políticas y normas públicas a nivel nacional e internacional \\
\cline { 2 - 3 } & Disponibilidad y facilidad de acceso a recursos físicos & T7 \\
\cline { 2 - 3 } & Situación del mercado y dinamismo del sector & T8 \\
\cline { 2 - 3 } & Relación oferta-demanda & Fuente: elaboración propia.
\end{tabular}

\section{Metodología}

Este estudio busca conocer cuáles son los elementos que han permitido que crezcan las startups de agrobiotecnología ganadoras de los concursos StartUp Perú y Reto Bio. A continuación, se detalla la metodología usada en la investigación.

\subsection{Estrategia de investigación}

El presente artículo pretende tener un panorama amplio y holístico al identificar los elementos clave de crecimiento de las startups estudiadas, por lo que se adopta una estrategia de investigación cualitativa (Taylor \& Bogdan, 1984; Hernández et al., 2010). De esta manera, se emplea un estudio de caso múltiple de tipo holístico, puesto que, por un lado, se analizan tres startups; y, por otro, cada caso se evalúa de forma integral (Yin, 2014). Asimismo, al tener un número acotado de casos, se presenta un cuadro resumen de los mismos para analizarlos luego, en el próximo apartado, a través de una selección de citas de los diferentes entrevistados (Beverland \& Lindgreen, 2010). En esa línea, para demostrar la calidad de la investigación, se ha optado por la fiabilidad, puesto que deja indicios de cómo se ha realizado la investigación a fin de que otros investigadores puedan replicarla y obtengan resultados similares (Berverland \& Lindgreen, 2010; Yin, 2018).

\subsection{Selección de los casos}

En el Perú se han dado iniciativas para el financiamiento de proyectos o emprendimientos relacionados a la agricultura, el ambiente y la biodiversidad, siendo uno de los más recientes el concurso Reto Bio, creado en el año 2016 por Innóvate Perú. Entre las categorías que este premio financia se encuentran los emprendimientos innovadores y dinámicos (Innóvate Perú, 2019). Asimismo, previamente, en el año 2012, se inició el concurso StartUp Perú que, que si bien no tenía por objetivo financiar únicamente proyectos con temáticas bio, ya ha financiado a diez startups de agrobiotecnología desde su tercera hasta la quinta generación. Ambas iniciativas cuentan con entidades de soporte como incubadoras y aceleradoras, de las cuales destacan Incubagraria y Bioincuba por su especialización en biotecnología (J. Álvarez, comunicación personal, 14 de octubre de 2019). 
Bajo ese contexto, entre los años 2015 y 2018 se han encontrado un total de 12 startups de agrobiotecnología (diez en StartUp Perú y dos en Reto Bio) que han participado y ganado en alguno de los concursos. Luego, la selección de la muestra se realizó en base a la conveniencia, debido a que fue factible acceder a tres startups (ver tabla 2), resultando de ello un muestreo no probabilístico (Hernández et al., 2010) y un estudio de múltiples casos (Yin, 2018). Asimismo, los casos seleccionados se consideran relevantes, puesto que tienen en promedio cuatro años en el mercado y su número de socios no asciende a cinco personas, excepto en el caso de Fertilizantes Muchik, que tiene personal adicional para la venta y trabajadores de planta.

\section{Tabla 2. Casos de estudio}

\begin{tabular}{|c|c|c|}
\hline Startup & Características & \\
\hline \multirow[t]{7}{*}{ Gervitro } & $\begin{array}{l}\text { Información } \\
\text { general }\end{array}$ & $\begin{array}{l}\text { - Fundada el } 2017 \text { en Arequipa y ganadora del concurso StartUp Perú 5G } \\
\text { - Número de socios: tres (incluido el CEO) }\end{array}$ \\
\hline & Producto & $\begin{array}{l}\text { Ornamento que contiene una planta en sus primeros estadios de } \\
\text { desarrollo y un gel que la mantiene con vida de } 6 \text { a } 12 \text { meses }\end{array}$ \\
\hline & $\begin{array}{l}\text { Propuesta de } \\
\text { valor }\end{array}$ & $\begin{array}{l}\text { Conservar, preservar y propagar especies en peligro de extinción } \\
\text { mediante soluciones biotecnológicas }\end{array}$ \\
\hline & $\begin{array}{l}\text { Relación con el } \\
\text { cliente }\end{array}$ & $\begin{array}{l}\text { - Gestión con clientes a través de redes sociales (se envía por courier) } \\
\text { - Soporte posventa a través de redes sociales y presencial }\end{array}$ \\
\hline & $\begin{array}{l}\text { Segmento de } \\
\text { clientes }\end{array}$ & $\begin{array}{l}\text { - Clientes: hombres y mujeres de NSE A, B o C de } 20 \text { a } 50 \text { años } \\
\text { - Usuarios: mujeres de } 16 \text { a } 45 \text { años que gustan de flores o regalos } \\
\text { novedosos }\end{array}$ \\
\hline & $\begin{array}{l}\text { Actividades } \\
\text { clave }\end{array}$ & $\begin{array}{l}\text { Investigación y desarrollo, gestión de proveedores, interacción con } \\
\text { clientes por redes sociales y asistencia a eventos para dar a conocer el } \\
\text { producto }\end{array}$ \\
\hline & $\begin{array}{l}\text { Información } \\
\text { financiera }\end{array}$ & $\begin{array}{l}\text { - Ingresos: venta de productos } \\
\text { - Costos y gastos: insumos, materiales de marketing para eventos, } \\
\text { trámites para patentar el producto, salarios e infraestructura }\end{array}$ \\
\hline \multirow[t]{7}{*}{$\begin{array}{l}\text { Fertilizantes } \\
\text { Muchik }\end{array}$} & $\begin{array}{l}\text { Información } \\
\text { general }\end{array}$ & $\begin{array}{l}\text { - Fundada el } 2014 \text { en Lambayeque y ganadora del concurso StartUp Perú } 3 G \\
\text { - Número de socios: cinco (incluido el CEO) }\end{array}$ \\
\hline & Producto & Fertilizantes elaborados a base de abonos orgánicos \\
\hline & $\begin{array}{l}\text { Propuesta de } \\
\text { valor }\end{array}$ & $\begin{array}{l}\text { Optimizar las tierras a través de productos de calidad, que cuiden el } \\
\text { medioambiente y no generen problemas para el bienestar del agricultor }\end{array}$ \\
\hline & $\begin{array}{l}\text { Relación con el } \\
\text { cliente }\end{array}$ & $\begin{array}{l}\text { - Primeros acercamientos vía redes sociales, correo electrónico y teléfono } \\
\text { - Gestión directa con los clientes }\end{array}$ \\
\hline & $\begin{array}{l}\text { Segmento de } \\
\text { clientes }\end{array}$ & $\begin{array}{l}\text { Empresas agroindustriales, empresas que buscan que se desarrollen } \\
\text { marcas, agricultores y asociaciones }\end{array}$ \\
\hline & $\begin{array}{l}\text { Actividades } \\
\text { clave }\end{array}$ & $\begin{array}{l}\text { Desarrollo del producto, comunicación del producto y manejo de la planta } \\
\text { de producción }\end{array}$ \\
\hline & $\begin{array}{l}\text { Información } \\
\text { financiera }\end{array}$ & $\begin{array}{l}\text { - Ingresos: venta de fertilizantes orgánicos } \\
\text { - Costos y gastos: insumos, infraestructura, salarios, capacitaciones y } \\
\text { servicios generales }\end{array}$ \\
\hline
\end{tabular}




\begin{tabular}{|c|c|c|}
\hline \multirow[t]{7}{*}{ Bioxlab } & $\begin{array}{l}\text { Información } \\
\text { general }\end{array}$ & $\begin{array}{l}\text { - Fundada el } 2016 \text { en Lima y ganadora del concurso Reto Bio } 2 \\
\text { - Número de socios: cinco (incluido el CEO) }\end{array}$ \\
\hline & Producto & Bioestimulante con levaduras inmovilizadoras de cadmio \\
\hline & $\begin{array}{l}\text { Propuesta de } \\
\text { valor }\end{array}$ & $\begin{array}{l}\text { Controlar los niveles de cadmio en las plantaciones, produciendo un } \\
\text { mayor rendimiento en los negocios }\end{array}$ \\
\hline & $\begin{array}{l}\text { Relación con el } \\
\text { cliente }\end{array}$ & $\begin{array}{l}\text { - Gestión directa con los clientes } \\
\text { - Seguimiento durante y después de la venta }\end{array}$ \\
\hline & $\begin{array}{l}\text { Segmento de } \\
\text { clientes }\end{array}$ & $\begin{array}{l}\text { Empresas, asociaciones o fundos medianos o grandes de palto, vid, } \\
\text { arándanos, cacao y espárragos }\end{array}$ \\
\hline & $\begin{array}{l}\text { Actividades } \\
\text { clave }\end{array}$ & $\begin{array}{l}\text { Investigación y desarrollo del producto, pruebas de validación en fundos, y } \\
\text { seguimiento durante la compra y posventa }\end{array}$ \\
\hline & $\begin{array}{l}\text { Información } \\
\text { financiera }\end{array}$ & $\begin{array}{l}\text { - Ingresos: venta de bioestimulantes orgánicos } \\
\text { - Costos y gastos: insumos, infraestructura, salarios, mantenimiento de } \\
\text { equipos y servicios generales }\end{array}$ \\
\hline
\end{tabular}

Fuente: elaboración propia.

\subsection{Recolección de la información}

En primer lugar, a partir de la revisión de literatura y entrevistas abiertas a especialistas en el tema, se elaboraron dos guías de entrevista semiestructuradas dirigidas a los CEO y los socios, respectivamente. Ambas guías se enfocaron en conocer aspectos generales de las startups, así como sus apreciaciones acerca del impacto que ha tenido una serie de elementos en su crecimiento. Para ello, cada una de las guías se estructuró en cinco partes: perfil del entrevistado, grupo del emprendedor, grupo del negocio, grupo del entorno y cierre. Además, para obtener mayor información de las startups estudiadas, se recurrió a información publicada en sus páginas web y redes sociales, así como a documentos internos de las mismas. Finalmente, a fin de validar los hallazgos, se elaboró una guía de entrevista para expertos en temas de startups y agrobiotecnología que tuvo la misma estructura que las anteriores. La lista de entrevistados se presenta a continuación.

\section{Tabla 3. Lista de entrevistados}

\begin{tabular}{l|l}
\multicolumn{1}{c|}{ Código } & Cargo e institución \\
\hline A1 & Investigador \\
\hline A2 & Docente PUCP \\
\hline A3 & Consultor de LF Gestión Total \\
\hline A4 & Presidente de Perubiotec \\
\hline A5 & Docente PUCP \\
\hline A6 & Director ejecutivo de CIDE-PUCP \\
\hline A7 & Docente PUCP \\
\hline S8 & CEO y gerente de Investigación y Desarrollo de Bioxlab S.A.C.
\end{tabular}




\begin{tabular}{l|l}
\hline S9 & Gerente de Operaciones de Bioxlab S.A.C. \\
\hline S10 & CEO de Gervitro \\
\hline S11 & Jefe de Producción de Gervitro \\
\hline S12 & Jefa de Laboratorio e Investigación de Gervitro \\
\hline S13 & Gestor de Negocios de Bioxlab S.A.C. \\
\hline S14 & CEO de Fertilizantes Muchik \\
\hline S15 & Asesora en Producción y Formulación de Bioxlab S.A.C. \\
\hline S16 & Administrador de Fertilizantes Muchik \\
\hline E17 & Directora técnica de Biotecnología Alimentaria S.A.C. \\
\hline E18 & Director general de la Dirección General de Información Agraria (DGIA) \\
\hline E19 & $\begin{array}{l}\text { Director de la Subdirección de Promoción de la Innovación Agraria del } \\
\text { INIA }\end{array}$ \\
\hline E20 & Consultor externo del CIP \\
\hline E21 & Docente PUCP \\
\hline G23 & Director de Iniciativa Empresarial de UPC \\
\hline
\end{tabular}

Fuente: elaboración propia.

\subsection{Proceso de análisis de la información}

Para analizar la información se utilizó la herramienta ATLAS.ti. En primer lugar, se transcribieron las entrevistas; luego, con la finalidad de sistematizar la información, se establecieron códigos a partir de la teoría de Vier (2016). De la misma forma, se crearon códigos adicionales para las variables empíricas que se encontraron en el trabajo de campo. Cabe resaltar que dichas variables fueron incluidas en los grupos del emprendedor, del negocio o del entorno según la implicancia de las mismas. Además, se analizaron los resultados, tomando en cuenta el contraste de los hallazgos con las entrevistas de validación a expertos. Por último, se constató la existencia de una heterogeneidad de perspectivas respecto al papel que cumplen los elementos en el proceso de crecimiento de las empresas, clasificándolas en tres rangos: alta (verde), media (amarilla) y poca o nula (roja). Para la división de estos elementos, se consideraron los siguientes aspectos: 1) ha estado presente en el proceso de crecimiento de la empresa y es percibido como clave por los emprendedores; y 2) los comentarios dados por los expertos respecto al papel que estos cumplen en el sector, la industria y el tipo de negocio en el que se encuentran los sujetos de estudio.

\section{Resultados y discusión}

\subsection{Elementos clave de crecimiento para startups de agrobiotecnología}

Como se detalló previamente, se agruparon los elementos clave de crecimiento de las startups de agrobiotecnología estudiadas en tres grupos: del emprendedor, del negocio y del entorno. 


\subsubsection{Dimensión del emprendedor}

\section{a. Nivel educativo y de especialización (E1)}

Este elemento hace alusión a que todos o, al menos, alguno de los miembros del equipo cuenten con conocimientos sobre biotecnología u otra ciencia relacionada. Por ejemplo, se identificó que en Gervitro los tres socios eran licenciados en Biotecnología, mientras que la CEO tenía una maestría en Ciencias Biomédicas. En Fertilizantes Muchik, por otro lado, dos de los cinco socios son licenciados en Biotecnología; y en Bioxlab, por su parte, cuatro de los cinco socios son licenciados en ciencias de la vida como la Biología. Asimismo, los integrantes de los equipos se han especializado en diversas temáticas, lo cual da como resultado una heterogeneidad de conocimientos y habilidades. De esta forma, se evidencia que contar con un nivel básico de biotecnología podría ser especialmente importante, de acuerdo al rol que desempeñe el colaborador.

\section{b. Conocimiento en negocios y gestión de empresas (E2)}

Los estudios o conocimientos en negocios y gestión de empresas terminan siendo relevantes para gestionar las demandas del negocio en los diferentes procesos clave y de soporte (Costa et al., 2004). Acerca de este elemento, en Gervitro lo han ido aprendiendo a medida que la startup crecía, pero como no era su especialidad y no tenían experiencia en ello, algunos temas — como la contabilidad y el marketing - fueron tercerizados o se contrató a alguien para ello. En Fertilizantes Muchik, por el contrario, dos de los socios ya tenían conocimientos de gestión de empresas, ya que eran licenciados en Administración; mientras que en Bioxlab solo uno de los socios tenía dichos conocimientos, por lo que el CEO y los otros socios empezaron a capacitarse en gestión, logística y producción.

En relación a los conocimientos adquiridos, los entrevistados resaltaron los beneficios de esta formación en tanto permite tener un mejor entendimiento de términos financieros y contables (S8), y de la gestión de los procesos internos de la empresa para la búsqueda de eficiencias (S9). Uno de los expertos resaltó la importancia de tener una base de conocimientos de gestión en los diferentes procesos del negocio, además de las ganas de emprender (E22). Esto no descarta el valor técnico que el emprendedor o su equipo pueden poseer en biotecnología y ciencias afines, sino que ambos elementos son complementarios y deben ser considerados en la gestión de este tipo de startups.

\section{c. Conocimiento en inversiones (E3)}

Este tipo de conocimiento es la base de la formulación de estrategias de inversión y expansión, y de la gestión de los recursos financieros y físicos de la empresa (Baptista et al., 2014). Para los entrevistados, este tipo de conocimiento abarca desde conocer el ecosistema de redes para saber lo que el inversionista busca (S13) hasta la gestión interna del capital de trabajo de la empresa (S16). Asimismo, a fin de formular estrategias de inversión, el equipo debe disponer de preparación y capacitación con los recursos que se tenga (S11). Sobre esto, en Gervitro, los socios no han tenido conocimientos previos en 
inversiones, pero están aprendiendo de manera autodidacta; en Bioxlab, solo uno de los socios tiene conocimiento del tema; y en Fertilizantes Muchik, los socios sí tienen estos conocimientos e incluso les han servido para captar posibles inversores.

Ante ello, uno de los expertos señaló que este conocimiento debe ir acompañado por la experiencia práctica en gestión de los recursos y el planteamiento de relaciones interinstitucionales para tener mayor claridad en la toma de decisiones y mantener un nivel mínimo de errores (E19). Además, el director general de la DGIA indicó que estos conocimientos pueden ser adquiridos por una especialización o cursos que permitan su desenvolvimiento a nivel teórico y práctico (E18).

\section{d. Liderazgo (E4)}

Se encontró que los entrevistados reconocen mayoritariamente a un único líder dentro de sus organizaciones, el cual usualmente tiene el cargo de CEO. De esta figura de líder destacan su rol como guía del negocio y su papel en la gestión del capital humano. Así, en Gervitro, como indicaron los entrevistados, el estilo inicial de su CEO no fue el ideal, pero hubo un desarrollo paulatino producto de la experiencia adquirida. En el caso de Bioxlab, su CEO es coordinado y participativo, además de apoyarse en uno de los socios; mientras que en Fertilizantes Muchik el CEO es reconocido como una persona capaz de gestionar la empresa y realizar networking. Para la gerente de Incubagraria, esta habilidad no debe ser desarrollada solo por el CEO, sino por todos los colaboradores a fin de que se puedan delegar funciones que conlleven tomar decisiones o liderar comunicaciones con clientes externos e internos como los proveedores, inversionistas y la sociedad cuando el CEO no pueda estar presente (E22). Asimismo, uno de los expertos mencionó que los líderes se adaptan a la situación de la organización y los requerimientos de su equipo (E20). Por lo expuesto, se valida el impacto del desarrollo de esta habilidad blanda en el proceso de toma de decisiones de la organización (Zucker et al., 1998) y, por consiguiente, su implicancia en el crecimiento de las startups.

\section{e. Experiencia y trayectoria (E5)}

En cuanto a la experiencia, normalmente se suele tomar en cuenta la experiencia en el puesto de trabajo y la experiencia en el sector de actividad. Para este caso, encontramos respuestas divergentes, ya que en Gervitro ninguno de los tres socios tenía experiencia ni en el puesto ni en el sector; en Fertilizantes Muchik, algunos de los socios tenían experiencia en el sector, mas no en el puesto; y en Bioxlab sucedía lo mismo que en Fertilizantes Muchik. Por tanto, se puede colegir que la experiencia y la trayectoria no necesariamente van de la mano; no obstante, tener una determinada experiencia podría ser relevante para una mejor toma de decisiones.

\section{f. Aspiración profesional y personal (E6)}

Teniendo un referente de rango temporal de tres a cuatro años, se encontró que los CEO y colaboradores presentan aspiraciones profesionales tanto dentro como fuera de la empresa, 
ya sea asumiendo nuevos roles o desarrollando nuevos conocimientos y habilidades mediante estudios de especialización, mayoritariamente realizados en el extranjero, en ciertos temas que pueden no estar relacionados directamente con su profesión. Cabe acotar que ambos puntos se direccionan a mejorar la situación actual de la empresa e innovar en sus productos. Ante ello, uno de los expertos indicó que dichas aspiraciones deben ser estimuladas y alineadas a los objetivos del negocio a fin de desarrollar una ventaja competitiva (E20). Por lo expuesto, se considera clave este elemento, ya que puede ser beneficioso por los nuevos conocimientos a adquirir y a ser implementados en la empresa; pero, como contraparte, hay un riesgo latente si es que el negocio no planifica la delegación de sus operaciones, teniendo en cuenta que la oferta de capital humano especializado es escasa. Esto último puede darse, por ejemplo, cuando una de las personas del equipo decida retirarse por un tiempo para seguir estudios fuera del país.

Por otro lado, con respecto a la aspiración personal, la cual se relaciona a la vida personal de los miembros del equipo, estas tienen una correlación con la edad en la toma de riesgos como parte de su proceso de crecimiento personal (Vier, 2016). Se halló que los entrevistados buscaban estabilidad económica y emocional, familia u objetivos personales. Además, en algunos casos, la aspiración profesional está alineada a la personal, pero en otros surge un conflicto para determinar cuál predominará. Uno de los expertos señaló que si bien tanto la aspiración profesional y la personal deben ir alineadas, no se debe perder de vista que son dos "cuerdas separadas» (E18) entre las cuales suele predominar la aspiración personal (E22). Por lo tanto, este elemento actúa activamente en la toma de decisiones del emprendedor, por lo que podría conllevar a contradecir las aspiraciones profesionales de los colaboradores en algún punto, razón por la que resulta importante considerarlo al momento de desarrollar estrategias de crecimiento y desarrollo del capital humano tanto a corto como a largo plazo.

\section{g. Valores y habilidades blandas}

Por un lado, entre los valores que los entrevistados enfatizaron se encuentran el compromiso y la honestidad. El primero se refiere al compromiso que el emprendedor presenta con el negocio y también el que demanda. La gerente de Incubagraria comentó que la evaluación de este elemento es relevante para determinar una visión clara y compartida en el negocio (E22). La honestidad que trasmiten los socios y CEO, por su parte, ha favorecido la construcción de relaciones con los agricultores y sus distribuidores, lo cual les ha servido para asegurar las ventas a corto y largo plazo (S16). Dicha relación la respalda uno de los entrevistados, quien mencionó que este valor es relevante para hacer crecer el negocio (S18).

Por otro lado, entre las habilidades blandas que los entrevistados resaltaron se encuentran la inteligencia emocional, la creatividad, la capacidad de innovación, la capacidad de negociación, el networking y la gestión del tiempo. De estas, la que destaca tanto para los entrevistados como para los expertos es el networking, puesto que, como indican los especialistas, es una gran fuente de información sobre agentes dispuestos a colaborar y a ayudar a la mejora de la empresa, y de potenciales alianzas estratégicas para temas específicos (E18, E19, E20 y E22). 
En suma, cada uno de los elementos de los valores y las habilidades blandas se relacionan entre sí y con otros factores ya descritos, además de desempeñar un papel clave según la etapa de crecimiento en la que se encuentre la startup.

\subsubsection{Dimensión del negocio}

\section{a. Educación y experiencia (N1)}

Por el lado del aprendizaje, los miembros de las startups estudiadas tienen diferentes niveles de estudio y especialización. Fertilizantes Muchik tiene colaboradores con nivel secundario y técnico (S14); Gervitro, socios y una colaboradora con licenciaturas; y Bioxlab, cuatro socios con maestría y uno con licenciatura. En lo que concierne a la experiencia, los miembros de las startups analizadas han tenido en algunos casos experiencia previa en el mercado laboral, la cual no necesariamente ha sido en el rubro, función o rol que actualmente asumen. Así, a pesar de que los miembros han sido inexpertos en ciertos temas al principio, han aprendido a lo largo del proceso de crecimiento sobre los puntos relevantes para la gestión de la organización, lo cual les sirve como experiencia para futuras decisiones. Esto valida que las organizaciones con más experiencia en el mercado tienen un mayor crecimiento, lo cual está relacionado con el aprendizaje y la edad (Teruel-Carrizosa, 2008).

\section{b. Conocimiento único (N2)}

Este elemento alude a una suma de conocimientos a nivel humano, organizacional y social que cumple un rol importante en el desarrollo de una ventaja competitiva (Subramaniam \& Youndt, 2005). Se consideró preguntar a cada uno de los colaboradores cuál sería, en primer lugar, el valor agregado que brindan a la empresa; y, luego, si este es sostenible en el tiempo —es decir, si es competitivo en el mercado-. Sobre esto, los entrevistados mencionaron como valor agregado la responsabilidad, la red de contactos, la creatividad, el conocimiento, la experiencia en el sector, la confianza y la honestidad. Ante ello, uno de los expertos indicó que el valor agregado principal es el conocimiento (E21), mientras que otro de los expertos consideró que las habilidades blandas eran relevantes (E22). En suma, los entrevistados resaltaron aspectos que tienen impacto en la manera en la cual se gestiona la organización y en cómo se desempeñan en el entorno, pero no rescatan el valor conjunto de todas estas habilidades, conocimientos e información externa e interna desarrollada, cuyo análisis y gestión permite formular una ventaja competitiva organizacional (Edvinsson \& Sullivan, 1996).

\section{c. Estrategia del capital humano (N3)}

Este elemento se refiere a los roles dentro del equipo y la potenciación de sus capacidades (Davidsson \& Honig, 2003). En primer lugar, se definieron los roles en función a los conocimientos, habilidades y/o experiencias que han desarrollado y adquirido antes y durante el inicio de operaciones de la startup. En Gervitro, ya que los tres socios tenían la 
misma formación en biotecnología y requerían profundizar en cómo empezar a exportar su producto, optaron por contratar a alguien para que les ayudase en ese tema. Fertilizantes Muchik, por su parte, al contar con un equipo multidisciplinario, pudo manejar la distribución de roles y contratar personas para las labores de planta y ventas. En Bioxlab, por último, también pudieron dividirse las funciones acorde a sus experiencias y conocimientos, pero con miras a contratar personas para que se encarguen de las ventas. Ante ello, algunos expertos comentaron que es necesario tener un "paraguas» de gente que conoce otras especialidades (E18), por lo que los emprendimientos deberían ser multidisciplinarios o, en su defecto, cada uno de los integrantes debe tener habilidades diferentes para que pueda haber una distribución eficiente de las tareas (E22).

Con respecto a la potenciación de capacidades, solo una de las tres startups estudiadas tiene un plan para ello; sin embargo, los miembros de las otras dos startups buscan por sí mismos talleres o cursos para desarrollar sus conocimientos y habilidades en determinados temas de interés (S8), o escogen a ciertos colaboradores para que reciban capacitaciones en determinados temas que la organización requiere que sepan (S16). Naturalmente, los métodos a implementar para potenciar las capacidades deben ser alineados a los recursos con los que actualmente cuenta la empresa (Kaiser \& Muller, 2015). Uno de los expertos mencionó que la capacitación y actualización de conocimientos y habilidades puede mejorar el desempeño del negocio (E18). En suma, resulta claro que la estrategia de capital humano va cumpliendo un papel clave a medida que surgen nuevas necesidades y requerimientos.

\section{d. Naturaleza o formato del negocio (N4)}

La naturaleza o formato del negocio se refiere al sector y modelo de negocio con el cual la empresa ha ingresado al mercado (Vier, 2016), lo cual incluye los riesgos y oportunidades que se presentan (Korunka et al., 2010). En este sentido, entre los riesgos que experimentan las startups estudiadas se encuentran la competencia, la imitación del producto, la poca facilidad para cambiar de proveedores de insumos y los riesgos operativos. Estos riesgos están siendo gestionados con la oferta de un servicio diferenciado y competitivo (S8), el aprovisionamiento de equipos de seguridad e instalaciones de estructura sólida (S14), la búsqueda de un nuevo proveedor por insumo y para un grupo de materiales, y la búsqueda constante de mejoras para diferenciar a su producto. Asimismo, uno de los expertos comentó que una startup de este tipo requiere de una alta inversión y corre el riesgo de tener un costo operativo alto si los productos no presentan la rapidez de venta requerida (E19). Además, en la biotecnología se presentan "muchos imponderables», ya que puede haber variaciones en productos como el cultivo in vitro, por ejemplo (E20). Por ello, debe considerarse la naturaleza o formato del negocio, ya que tiene influencia en el financiamiento y la demanda.

\section{e. Gestión de recursos financieros (N5)}

Este elemento se operativiza con fuentes de financiamiento y planes financieros. Sobre el primer factor, los entrevistados hicieron uso de los fondos concursables de StartUp 
Perú y Reto Bio, e incluso de otros como Ideas Audaces de Concytec. Además, a fin de reducir costos, están evaluando aliarse con proveedores o distribuidores. Algunos expertos afirmaron que debido a que las inversiones en biotecnología son costosas, las startups deberían contar con alguna planificación de cómo se va a gestionar el financiamiento a corto y a mediano plazo (E18), lo cual podría financiarse con recursos propios (E21) antes de que las startups puedan sustentarse con fondos concursables. Para poder continuar se requiere una proyección destinada a optimizar los gastos e inversiones a realizar; por tanto, se considera clave tener presente una estrategia de financiamiento.

\section{f. Relación con proveedores (N6)}

Las asociaciones o alianzas con los proveedores pueden influir en el crecimiento de las ventas (Beekman \& Robinson, 2004). Por ello, algunos entrevistados señalaron que se encuentran elaborando una lista de proveedores a fin de encontrar los mejores precios y calidad asegurada (S9). Por el contrario, una de las startups tiene inconvenientes para la obtención de insumos, puesto que dependen de proveedores únicos para varios de ellos que están localizados en diferentes lugares, lo cual ha provocado que algunos de sus proveedores quieran aprovecharse y cobrar un precio adicional (S10). En relación a esto, uno de los expertos indicó que es necesaria la gestión de proveedores ya que estos pueden ser muy especializados, por lo cual sería ideal conseguir más de uno para cada insumo (E23).

\section{g. Gestión de la innovación (N7)}

Este elemento alude al proceso por el cual se mejoran o crean nuevos productos, procesos, formas de comercializar y de realizar cambios organizativos para adaptarse al entorno y generar ventajas competitivas sostenibles en el tiempo que le permitan a la startup asegurarse en el mercado (Seclen, 2016). Todos los entrevistados mencionaron que están en constante búsqueda de mejoras y oportunidades de desarrollo de nuevos productos. Para este proceso, Fertilizantes Muchik cuenta con un equipo dedicado a ello, mientras que en Gervitro y Bioxlab la innovación se adhiere como una función más dentro de los roles de todos. Frente a ello, los expertos opinaron que la innovación requiere de investigación constante y rigurosa, además de conocimiento especializado en biotecnología y en temáticas relacionadas como la biología y microbiología (E18); por lo mismo, se requiere una gran inversión, pero esta puede garantizar una oportunidad de crecimiento a la empresa (E20).

\section{h. Estrategia comercial}

Este es uno de los elementos encontrados en el trabajo de campo, y comprende la gestión de marketing-comercial y la fuerza de ventas. Con respecto a la primera, el CEO de Bioxlab indicó que al principio no se enfocaron en la parte comercial, pero ahora sí le están dando más importancia, puesto que parte de sus objetivos es aumentar sus ventas y optimizar la dosificación de su producto para hacerlo más atractivo (S8). En el caso de Gervitro, sus ventas se realizan por canales digitales y actualmente han identificado una oportunidad 
para exportar sus productos. Fertilizantes Muchik, al igual que Bioxlab, también se está enfocando en la parte comercial y en lograr una mayor difusión de su producto (S16). Al respecto, los expertos comentaron que es fundamental contemplar la parte comercial para salir al mercado, pero este tipo de emprendimientos no suele hacerlo pues las personas involucradas se dedican más a la investigación (E22), por lo que debe desarrollarse la habilidad para hacer visible el producto (E19).

Con respecto a la fuerza de ventas, Bioxlab resaltó dicha necesidad, puesto que además de explicar el producto al cliente y efectuar la venta, hay otros conocimientos que requieren de la fuerza de ventas, como la dosificación del producto en campo. Esto guarda relación con lo mencionado por uno de los expertos, quien precisó que si el equipo no tiene conocimiento sobre algún tema, debería optar por contratar a alguien que brinde el servicio o busque alianzas (E22). Después de lo explicado, tanto la gestión de marketing-comercial como la fuerza de ventas pueden considerarse claves, ya que apuntan hacia la generación de mayores ingresos y al desarrollo de ciertos conocimientos.

\section{i. Gestión de la cadena de suministros}

Este es un elemento que se encontró durante la fase de campo y se puede definir como la gestión o manejo para que los productos se distribuyan de manera directa o indirecta. Los entrevistados, en especial Bioxlab y Gervitro, enfatizaron la importancia de este elemento; e incluso esta última comentó que tenía problemas para trasladar sus productos, ya que a veces estos no llegaban en buen estado a sus clientes. Por su parte, Fertilizantes Muchik no presentaba inconvenientes debido a que su planta se encontraba cerca de sus clientes objetivo. En referencia a esto, uno de los expertos indicó que es conveniente buscar canales de comercialización del producto, pero esto corresponde al escalamiento de la empresa (E21). En ese sentido, como las startups estudiadas todavía están en crecimiento, necesitarán ampliar paulatinamente su capacidad de producción y distribución, por lo cual debe considerarse cómo se gestionarán para satisfacer las necesidades de sus clientes con la mayor eficacia posible.

\subsubsection{Dimensión del entorno}

\section{a. Clúster (T1)}

Este elemento es considerado nulo, debido a que no se ha identificado aún su participación en el Perú. Sin embargo, los emprendedores consideran que hay potencial para que un clúster se desarrolle (S8) y, además, hay disposición por parte de las startups a ser parte de uno si es que está relacionado al producto que comercializan (S16). Para que esto se concrete, se requieren acciones que alineen a los actores privados y públicos (E21) (Moodysson et al., 2006).

\section{b. Venture capital e inversionistas ángeles (T2)}

Ambos elementos son importantes, ya que sin ellos se presenta un mayor número de obstáculos al desarrollo del ecosistema emprendedor (Peris, 2014); sin embargo, a pesar 
de ello, las startups analizadas no han recibido o concretado un acuerdo de financiamiento con estos actores. Según los emprendedores, hay un número limitado de inversionistas que conocen el tema, tal como han podido extraer del contacto mantenido con estos actores. En esa línea, los expertos indican que las participaciones de los inversionistas se deben dar en etapas como la del crecimiento (E22) y que, efectivamente, la inversión en este tipo de startups implica mucho riesgo (E19).

Debido al rol similar que cumple el venture capital, este debe aparecer en etapas intermedias; es decir, en cuanto la startup tenga un posicionamiento en el mercado que le genere ventas suficientes para autosostenerse, ya que esto aminora el riesgo de inversión y presenta mayores probabilidades de atraer inversionistas para este tipo de emprendimientos.

Por otro lado, según las startups estudiadas, se tuvo contacto con inversionistas ángeles que no se concretaron (S15). Esto se dio, por un lado, por la dificultad de trasladarse a la capital (Lima) (S16); y, por otro, pues no identificaron a un inversionista que los pudiera apoyar apropiadamente por desconocimiento del negocio (S11). Según los expertos, al tener contacto con alguno de estos inversionistas, se recomienda sensibilizar sobre este tipo de tecnología (E22), ya que dichos actores aparecen principalmente en las primeras etapas (Peris, 2014). Por último, se recomienda presentar en términos económicos y técnicos la viabilidad de la idea (E21).

En resumen, este tipo de startups no solo busca financiamiento, sino también una fuente de información y de contactos estratégicos para impulsar el crecimiento de la empresa, y un inversionista que conozca el sector y nicho de mercado en el que se encuentran (S11). Dicho esto, se puede afirmar que cumplen un papel clave en el crecimiento de esta clase de startups.

\section{c. Universidades y centros de investigación (T3)}

La perspectiva sobre las universidades se clasifica en dos frentes: por un lado, como agentes de apoyo y soporte (S16) por los recursos que ofrecen (S13); y, por otro, se las considera sistemas burocráticos que expanden el tiempo para nuevos desarrollos y limitan el crecimiento de la startup (S11), así como la calidad del servicio brindado (S14). Según los expertos, estos agentes cumplen un rol estratégico mediante la oferta y el constante desarrollo de conocimientos y recursos (E18). El valor agregado de ello se refleja en el proceso de innovación que surge de aplicar nuevas tecnologías y al facilitar el acceso al conocimiento mediante asesorías (E21) u otros medios (Seclen \& Barrutia, 2019; Tostes, 2014).

Por otro lado, los centros de investigación actúan como promotores del desarrollo y de los productos que impactan positivamente en el crecimiento económico de los agricultores (E19), y se diferencian de las universidades por la especialización que tienen en estos temas, y también por sus niveles de financiamiento, que permiten la investigación y desarrollos (E20).

Ambos actores se alinean con las estrategias que el sector ha planteado para incentivar la aplicación de esta tecnología (Concytec, 2016); sin embargo, según los expertos, es importante que se identifique o se estructure un centro de investigación que tenga como foco la biotecnología o el bioemprendimiento (S10) para facilitar la interacción entre los actores de interés (S11). 
Las startups identificaron un número de incentivos fiscales; no obstante, no todos aplicaban a su tipo de emprendimiento (S13). Otros, como un menor porcentaje de IGV, facilitan la venta de productos al permitirles tener un precio competitivo (S16). Según los expertos consultados, otros beneficios, como un menor pago de impuestos, también impactan en el crecimiento de las startups, principalmente en las primeras etapas (E20). Sin embargo, otros mencionan que el impacto sería menor —es decir, leve- en el crecimiento de una startup (E19). Por otro lado, hay expertos que comentan que normas como la Ley №30309, que promueve la investigación científica, deben ser evaluadas para extenderlas al sector agrario debido a los beneficios que esto brindaría a las startups (E18).

En conclusión, se presentan perspectivas diferentes respecto al impacto o relevancia que los incentivos fiscales tendrían en este tipo de startups. En otros estudios se identificó que este elemento tenía impactos positivos en el crecimiento (Menrad, 2000) y sobre el número de startups presentes en el mercado (Newell, 2007). Esto se debe a que las startups tendrían la posibilidad de destinar una mayor inversión a la innovación, a optimizar sus operaciones o incluso al ámbito comercial, los cuales son puntos estratégicos para el crecimiento.

\section{e. Políticas y normas públicas a nivel nacional e internacional (T5)}

Según las startups analizadas, las políticas públicas del país no promueven o favorecen a las startups de agrobiotecnología, ya que estas no aclaran ni especifican temas como la explotación de plantas y los procesos aduaneros relacionados a los productos que comercializan (S10, S14). Por otro lado, la promulgación y el seguimiento de las políticas también deben ser reforzados en los nichos donde actualmente se encuentran las startups estudiadas como el de biofertilizantes, segmento en el que se presenta informalidad y poca fiscalización en los procesos, lo cual pone en peligro la sostenibilidad de los cultivos (S16). Asimismo, para la mayoría de las startups se han presentado inconvenientes en los trámites para adquirir una patente de sus productos (S8, S10).

Según los expertos, las normas o leyes peruanas no son precisamente adecuadas ni dan facilidades debido a que no están alineadas a la aplicación de la biotecnología; es decir, han quedado obsoletas (E22). Además, las normas internacionales que se han firmado limitan el desarrollo o innovación en determinados rubros, por lo que se propone evaluar su renovación a fin de determinar su impacto en el mercado de la biotecnología (E18).

En resumen, si bien las políticas nacionales e internacionales actúan como barreras, también pueden ser impulsadoras del desarrollo del mercado de biotecnología, dependiendo de la aplicación de los productos; no obstante, en Perú predomina lo primero. Debido al impacto que la políticas generan, tanto las startups como los entrevistados consideran a este elemento como uno clave para el crecimiento (OCDE, 2015).

\section{f. Disponibilidad y facilidad de acceso a recursos físicos (T6)}

Los emprendedores de las startups estudiadas mencionaron que han requerido o dispuesto de recursos tangibles, como ambientes de trabajo (S10) y equipos especializados 
(S13), como parte de los premios de los concursos en los cuales participaron y ganaron (S14). En el caso de aquellos recursos a los cuales no tuvieron acceso o disponibilidad, los emprendedores los adquirieron por su cuenta y tomando en consideración los límites geográficos y financieros (S11, S16). De este último punto, se debe resaltar este tipo de barreras para determinados materiales requeridos por los laboratorios de biotecnología (E18). Por ello, se recomienda evaluar otros medios o canales para poner a disposición los recursos que presentan estas limitaciones y, a la par, cuestionar su viabilidad de adquisición y sus lineamientos de uso.

\section{g. Situación y dinamismo del mercado (T7)}

Sobre este elemento, los hallazgos se dividen en cuatro pilares importantes: sector, línea de productos, tecnología aplicada y capital humano. Del primero, los entrevistados señalaron que el mercado está cada vez más competitivo debido a una mayor demanda de productos que apliquen biotecnología, dados los beneficios que esta brinda (S8, S16, S15). Del segundo punto, cabe señalar que los productos que cada startup brinda no son de alto riesgo en términos de uso, pero sí requieren de un nivel de inversión alto para cubrir el costo de la maquinaria y los materiales a utilizar (S11, S16, E18). Según los expertos, se recomienda tener una estrategia de sensibilización al momento de realizar la venta de los productos, pues no necesariamente todos conocerán los beneficios de la biotecnología (E22) o el concepto con el cual se ha estructurado el producto (A20).

Del tercer punto, las startups analizadas comentaron que es complejo por el tipo de formación aplicada y la especialidad que se requiere de acuerdo a los diferentes focos de la biotecnología (S12, E19, E20, E17); competitivo, por el constante cambio y las novedades que presenta (S9, S12, E18); y de desarrollo lento, si se compara con otros países de Latinoamérica (S10, S15, E19). Por último, existe un número limitado de especialistas con ese perfil determinado en el mercado, y son principalmente aquellos que integran la formación en comercio y biotecnología (S8) (Concytec, 2016).

En resumen, el mercado cumple un rol de alta criticidad también para el crecimiento de este tipo de startups, ya que la aceptación que el público objetivo presente sobre los productos determinará su continuidad y escalabilidad. Por ello, es importante tomar en consideración las variables anteriormente mencionadas, y evaluar el costo de oportunidad que estas involucran y si realmente son viables en el mercado analizado.

\section{h. Relación entre oferta-demanda (T8)}

De acuerdo al análisis de los productos que ofrecen las startups, se concluye que hay nichos de mercado específicos con una frecuencia de demanda alineada a las necesidades del cliente y que consideran los riesgos y retos que estos representan (Menrad, 2000).

Sobre esta oferta, se han presentado comentarios divididos y alineados al contexto o la geografía de los clientes, como el formato en el que se vende el producto y si este requiere de determinados insumos escasos en determinadas zonas (S15). Por el lado positivo, se valora cómo se brinda el servicio — por ejemplo, si es personalizado- y 
el servicio posventa, incluyendo factores como las visitas frecuentes durante el periodo de prueba del producto en determinados cultivos (S9).

Por otro lado, algunas startups han implementado canales digitales para facilitar la venta de sus productos e incluso han traspasado fronteras al obtener demanda internacional (S14). Pese a ello, dichas oportunidades no se concretaron en una venta por límites impuestos por las políticas aduaneras (S11).

\section{i. Red de startups, de información y de emprendedores}

Según expertos, el uso de diferentes canales de venta y de exposición del producto es importante (E19), ya que favorecen al emprendedor en el proceso de identificar nuevos mercados (E21) y oportunidades de benchmarking (E22). De estos mecanismos, se identificaron los siguientes: una red de startups y emprendimientos, una red de emprendedores y, por último, una red de información centralizada y de soporte. De este último elemento, se precisa que su papel tendría mayor impacto en las primeras etapas del proceso de crecimiento de la startup por la información que brindará (E20).

\section{j. Red internacional e incubadoras}

En la etapa de campo, se relevó información sobre dos elementos clave considerados por los entrevistados: la relación con incubadoras y las redes internacionales de biotecnólogos y biotecnología. Del primer elemento, se rescatan casos de apoyo por parte de determinadas incubadoras pertenecientes al sector público en Lima (S9) y Arequipa (S10). Sin embargo, se precisó que este apoyo presentó un número de limitantes, como el hecho de no estar enfocado en este tipo de startups en particular (S10). Según los expertos en el tema, el papel de este actor es clave y es una prioridad reforzar los conocimientos en estos temas debido al tipo de asesoramiento que requiere esta clase de startups en particular (E22).

En el caso del segundo elemento, se identificó que una de las startups analizadas formaba parte de una de estas redes, conocida como AllBiotech. Según comenta, al pertenecer a esta red, se ha tenido mayores oportunidades de adquirir y explotar conocimiento, ganando experiencia (S10). A la par de ello, esto ha permitido a esta startup posicionarse en Latinoamérica al asistir a foros y ferias (S12). Según los expertos, el papel de estas redes es, como en el caso de las incubadoras, relevante por su contribución a fomentar el emprendimiento en este sector, aplicando esta clase de tecnología (E20).

En síntesis, hay 21 elementos que las startups estudiadas consideran clave y están incluidos dentro de la teoría, mientras que en el trabajo de campo se pudieron rescatar seis elementos adicionales (ver figura 1). 
Figura 1. Elementos clave de crecimiento de las startups de agrobiotecnología estudiadas

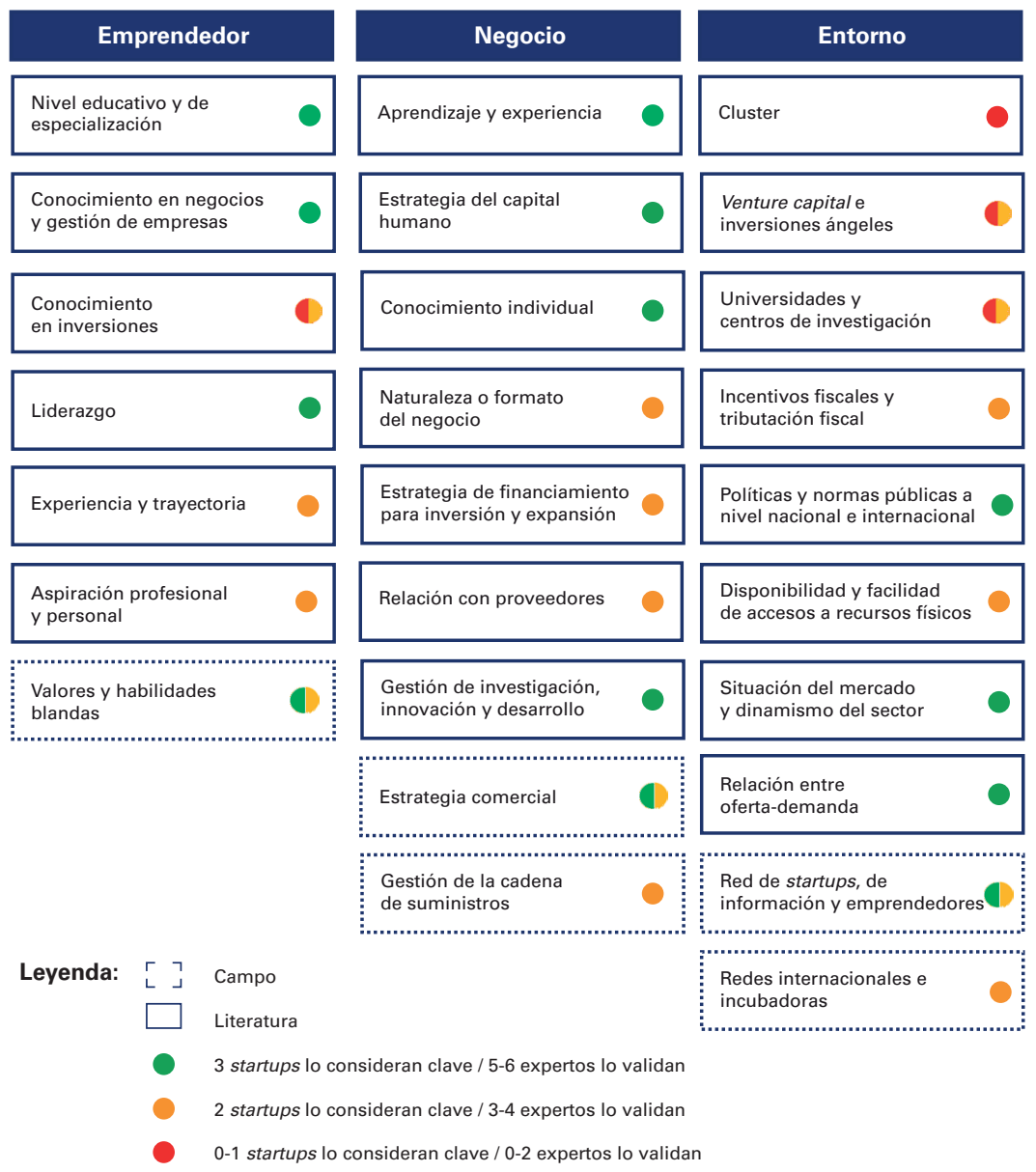

Fuente: elaboración propia.

\subsection{Clasificación de crecimiento de las startups seleccionadas}

Dado que uno de los filtros de selección de la muestra que se consideró fue que las startups estuvieran en etapa de crecimiento, se les clasificó según el modelo de Hernández y González (2016), pero complementado con 11 categorías del modelo de Scott y Bruce (1987), las cuales son la etapa de la industria, los principales problemas, el rol y el estilo de la gestión, la estructura organizacional, la investigación de mercado y de producto, los sistemas y controles, la principal fuente de financiamiento, la generación de dinero, las principales inversiones, el producto y el mercado.

Teniendo en cuenta ello, se determinó que, según el modelo de Hernández y González (2016), Bioxlab y Fertilizantes Muchik se encontraban en la etapa de crecimiento, mientras que Gervitro estaba entre las etapas de eficiencia y crecimiento. Finalmente, desde el análisis con el modelo de Scott y Bruce (1987), se halló que Bioxlab estaba en la etapa de crecimiento; Gervitro, entre las etapas de sobrevivencia y crecimiento; y Fertilizantes Muchik, entre las etapas de crecimiento y expansión. 


\section{Conclusiones}

El objetivo de la presente investigación fue conocer los elementos clave de crecimiento de las startups de agrobiotecnología en el Perú ganadoras de los concursos Startup Perú o Reto Bio, identificando y describiendo dichos elementos y sus respectivos vínculos con el crecimiento de cada startup y la forma en que lo han influenciado. Este estudio revela que las startups de agrobiotecnología peruanas tienen un número de elementos con alto potencial de desarrollo para viabilizar un mayor escalamiento de las mismas a nivel internacional; sin embargo, estas requieren aún de acciones orientadas a fomentar las diferentes dimensiones del crecimiento, ya que se presentan determinadas barreras en cada una de ellas que dificultan el escalamiento en determinados puntos del Perú. Entre estas se encuentran los procesos burocráticos y no tan eficientes de formalización de patentes y exportación de este tipo de productos, además de la centralización de los recursos financieros y físicos disponibles para las startups debido a la particularidad de la industria y el mercado.

De la identificación de la etapa de crecimiento de las startups se concluye lo siguiente: dos de las tres startups estudiadas se encuentran en la etapa de crecimiento según Hernández y González (2016); la tercera se encuentra en transición de la etapa de eficiencia a la de crecimiento. Esto se debe a una variable principal: que aún no ha alcanzado el punto de equilibrio en ventas que les permita reinvertir en el negocio. En otras palabras, no cuenta con el capital necesario para ejecutar sus planes de expansión; sin embargo, se encuentran en búsqueda del mismo para proceder a implementar los planes previamente analizados.

El estudio identificó 21 elementos clave de crecimiento a partir del marco teórico; luego, en la fase de campo, se agregaron cinco elementos, dando un total de 26 . En la etapa de validación, diez de estos elementos se consideraron claves para el crecimiento de las startups, pues no necesariamente todos han estado presentes en el proceso de crecimiento de cada una de las startups; y, además, se tomó en cuenta la validación de expertos en el rubro.

Entre otras conclusiones, en el primer grupo — catalogado como en el del emprendedor - se rescata que no se debe presentar un nivel educativo o de especialización homogéneo, pero se valida que el equipo debe tener conocimientos básicos de biotecnología o de ciencias derivadas y de negocios para escalar en este determinado sector (Zucker et al., 1998; Staniewski, 2016; Dorockis \& Bogus, 2014), debido a los roles y funciones que los emprendedores asumen según el crecimiento del negocio (Costa et al., 2004). Esto no descarta que se requiera de profesionales con mayor experiencia en el rubro o en la tecnología que utiliza la empresa, pues estos han estado presentes como profesores de universidades y/o consultores brindados por las incubadoras $u$ otras instituciones. Por otro lado, se identificó que los conocimientos adquiridos deben ser diversos —es decir, no solo en un área o línea de carrera- para favorecer las etapas de innovación (Oe \& Mitsuhashi, 2013), el planteamiento de estrategias comerciales (Seclen \& Barrutia, 2019), etc. Asimismo, entre los conocimientos adquiridos se consideran aquellos que se han obtenido a lo largo del proceso de emprendimiento y crecimiento del negocio (Hernández \& González, 2016). En esa línea, de los elementos identificados en la fase 
de campo, los conocimientos aprendidos se presentan principalmente en el elemento de valores y habilidades blandas, dentro del cual destaca el networking debido a los beneficios y oportunidades que deriva para el negocio a nivel nacional e internacional.

Del grupo del negocio se resalta el papel del elemento de gestión de la innovación por el rol que cumple con los objetivos de la empresa. En este caso, se rescata que es un proceso particular, ya que está alineado a las bases de la investigación científica; es decir, es metódica, precisa y con pasos de prueba-error. Por ello, es importante considerar la inversión que estas startups requieren tanto a nivel financiero como de tiempo, particularmente para productos con fines específicos. A pesar de todo ello, este proceso ha beneficiado a las startups al extender su línea de productos con apoyo de su estrategia comercial y de la relación con sus clientes. En otras palabras, a pesar de que suene poco rentable al comienzo, es un sector que tiene alto potencial en innovación de productos (Honjo \& Nagaoka, 2018). Por otro lado, se recomienda a las startups evaluar la gestión de sus proveedores debido a que esta podría impactar en su escalabilidad como negocio y sobre sus costos a largo plazo.

En el grupo del entorno se identificó que los elementos que presentan un rol prioritario son la disponibilidad de recursos físicos, las políticas a nivel nacional e internacional, y los centros de investigación y universidades. Del primer elemento, se rescata que puede limitar o promover a las startups del rubro según el enfoque que abarquen, lo cual se refleja en países como India y China. De las universidades, se rescata que son fuentes de conocimiento, pero según las startups y expertos, estas deben optimizar sus procesos en la gestión de aquellos proyectos que fomentan y dar soporte a emprendimientos, principalmente de este tipo. No obstante, lo recién dicho no las desestima, y tampoco a los centros de investigación, como potenciales aliados estratégicos debido a los recursos tangibles e intangibles que aportan y al capital humano que proveen. Entre los elementos de campo identificados, se determinó que no solo buscan aumentar la representatividad de estas startups en el mercado, sino también fomentar la bioeconomía en los mercados desde una perspectiva global al incentivar el contacto con casos de éxito a nivel nacional o en países vecinos.

Por otro lado, se concluye que los elementos presentan diferentes tipos de relaciones entre sí o, en algunos casos, ningún tipo de relación directa. Se debe precisar que ningún grupo se sobrepone a otro, ya que cada uno de ellos y sus elementos respectivos cumplen un rol determinado en la etapa de crecimiento de la startup, el cual no necesariamente va alineado a los objetivos o estrategias de la startup resultado de las interacciones. En otras palabras, no todos los elementos presentan un rol de facilitadores del crecimiento y se requiere una mayor magnitud de análisis sobre su impacto en las decisiones de las startups por su complejidad en el mercado.

Por último, en relación al estudio, debido a la estrategia metodológica aplicada, los hallazgos anteriormente mencionados no se pueden generalizar, por lo que se recomienda realizar estudios cuantitativos para validarlos. Además, dado que se realizó un estudio de los elementos a nivel macro, se recomendaría profundizar en uno de ellos: el de gestión de la innovación. Estos estudios tendrían un mayor valor agregado si se realizaran con startups de diferentes partes del Perú para rescatar sus particularidades a nivel nacional. 


\section{bibliografía}

Aguinaga, R.

Biotecnología: Visión General. Revista de Química, 2(1), 41-45. Recuperado de http:// revistas.pucp.edu.pe/index.php/quimica/ article/view/4862

\section{Baptista, R., Karaöz, M., \&} Mendonça, J.

The impact of human capital on the early success of necessity versus opportunitybased entrepreneurs. Small Business Economics, 42(4), 831-847. https://doi. org/10.1007/s11187-013-9502-z

\section{Beekman, A., \& Robinson, R.}

Supplier Partnerships and the Small, High-Growth Firm: Selecting for Success. Journal of Small Business Management, 42(1), 59-77. Recuperado de https://sci-hub. tw/10.1111/j.1540-627x.2004.00097.x

\section{Beverland, M., \& Lindgreen, A.}

What makes a good case study? A

positivist review of qualitative case research published in Industrial

Marketing Management, 1971-2006.

Industrial Marketing Management,

39(1), 56-63. https://doi.org/10.1016/j. indmarman.2008.09.005

\section{Blank, S., \& Dorf, B.}

The Startup Owner's Manual: The Step By Step Guide for Building a Great Company. California: K \& S Ranch.

Canals, J.

Crecimiento empresarial: personas y tecnología en la nueva economía. Revista Empresa y Humanismo, 2(2), 337-370.
Capelleras, J. L., \& Kantis, H. D.

El fenómeno del crecimiento de las nuevas empresas. En Joan-Lluís Capelleras Segura (ed.), Nuevas empresas en América Latina: factores que favorecen su rápido crecimiento (pp. 11-20). Barcelona: Universitat Autónoma de Barcelona. Recuperado de https://colaboracion.dnp. gov.co/CDT/Desarrollo\%20Empresarial/ Nuevas \%20empresas \%20en \% 20 America\%20Latina_factores \%20que \% 20 favorecen $\% 20$ su $\% 20$ crecimiento $\% 20$ (2009).pdf

\section{Comisión Económica para}

América Latina y el Caribe (CEPAL), Food and Agriculture Organization (FAO), \& Instituto Interamericano de Cooperación para la Agricultura (IICA)

Perspectivas de la agricultura y del desarrollo rural en las Américas: una mirada hacia América Latina y el Caribe 2019-2020. San José: IICA. Recuperado de https://repositorio.iica.int/bitstream/ handle/11324/8214/BVE19040295e. pdf? sequence $=1$

\section{Consejo Nacional de Ciencia,} Tecnología e Innovación Tecnológica (Concytec)

Programa Nacional Transversal de Biotecnología 2016-2021. Recuperado de https://portal.concytec.gob.pe/images/ noticias/PRONBIOTEC_FINAL.pdf

\section{Consejo Nacional del Ambiente} (CONAM)
El desarrollo biotecnológico en el Perú.

Lima: CONAM 


\section{bibliografía}

Costa, C., Fontes, M., \& Heitor,

M. V.

2004

A methodological approach to the

marketing process in the biotechnology-

based companies. Industrial Marketing

Management, 33(5), 403-418. Recuperado

de https://www.sciencedirect.com/science/

article/abs/pii/S0019850103001184

\section{Davidsson, P., \& Honig, B.}

2003 The role of social and human capital among nascent entrepreneurs. Journal of Business Venturing, 18(3), 301-331. https:// doi.org/10.1016/S0883-9026(02)00097-6

\section{Dorockis, S., \& Bogús, M.}

2014 Regional Variety of Biotechnology

Development in Asia. Procedia - Social

and Behavioral Sciences, 1(20), 197-212.

https://doi.org/10.1016/j.sbspro.2014.02.097

\section{Edvinsson, L., \& Sullivan, P.}

Developing a model for managing intellectual capital. European Management Journal, 14(4), 356-364. https://doi. org/10.1016/0263-2373(96)00022-9

\section{Gertler, M. S., \& Levitte, Y. M.}

Local Nodes in Global Networks: The Geography of Knowledge Flows in Biotechnology Innovation. Industry and Innovation, 12(4), 487-507. Recuperado de https://www.tandfonline.com/doi/ full/10.1080/13662710500361981

\section{González, R.}

Biotecnología, Historia y Desarrollo:

Situación Actual en Nicaragua. En //

Congreso Multidisciplinario e Internacional de Agrobiotecnología [Ponencia]

Recuperado de https://www.researchgate.
net/publication/274138026_Biotecnologia_ Historia_y_Desarrollo_Situacion_Actual_ en_Nicaragua

\section{Hernández, R., Fernández C., \& Baptista P.}

Metodología de la investigación (5ta edición). México: McGraw Hill.

\section{Hernández, C., \& González, D.}

2016 Study of the Start-Up Ecosystem in

Lima, Peru: Collective Case Study. Latin American Business Review, 17(2), 115-137. https://doi.org/10.1080/10978526.2016.11 71678

Hidalgo, G., Kamiya, M., \&

\section{Reyes, M.}

Emprendimientos dinámicos en América Latina: Avances en Prácticas y Políticas. Caracas: Corporación Andina de Fomento. Recuperado de http://scioteca.caf. com/bitstream/handle/123456789/371/ emprendimientos_dinamicos_america_ latina. pdf? sequence $=1$ \&isAllowed $=y$

\section{Honjo,Y., \& Nagaoka, S.}

Initial public offering and financing of biotechnology start-ups: Evidence from Japan. Research Policy, 47(1), 180-193. https://doi.org/10.1016/j.respol.2017.10.009

\section{Innóvate Perú}

$$
\begin{aligned}
& \text { Programa Nacional de Innovación para } \\
& \text { la Competitividad y Productividad- } \\
& \text { Innóvate Perú [PPT]. Recuperado de http:// } \\
& \text { innovacion.concytec.gob.pe/wp-content/ } \\
& \text { uploads/2019/07/expositora_2-rosmary- } \\
& \text { cornejo-valdivia.pdf }
\end{aligned}
$$




\section{bibliografía}

Kaiser, U., \& Müller, B.

2015

Skill heterogeneity in startups and its

development over time. Small Business

Economics, (45), 787-804. https://doi.

org/10.1007/s11187-015-9667-8

Korunka, C., Kessler, A., Frank, H., \& Lueger, M.

2010

Personal characteristics, resources, and

environment as predictors of business

survival. Journal of Occupational and

Organizational Psychology, 83(4),

1025-1051. Recuperado de https://www. researchgate.net/publication/229786637_

Personal_characteristics_resources_and_ environment_as_predictors_of_business_ survival

\section{Menrad, K.}

2000

Anniversary Review Economic Implications of Agro-food biotechnology. Journal of the Science of Food and Agriculture, (80), 539-546. Recuperado de https:// onlinelibrary.wiley.com/doi/10.1002/

(SICI)1097-0010(200004)80:5\%3C539::AIDJSFA564\%3E3.0.CO;2-1

\section{McKelvie, A., \& Wiklund, J.}

Advancing firm growth research: A focus on growth mode instead of growth rate. Entrepreneurship Theory and Practice, 34(2), 261-288.

\section{Moodysson, J., Ryan, C. D.,} Asheim, B., \& Phillips, P.

Comparing a Pharmaceutical and an

Agro-food Bioregion: On the Importance

of Knowledge Bases for Socio-spatial

Patterns of Innovation. Industry and

Innovation, 13(4), 393-414. Recuperado

de https://www.tandfonline.com/doi/

full/10.1080/13662710601032937
Moroni, I., Arruda, A., \& Araujo, K.

2015

The Design and Technological

Innovation: How to Understand the

Growth of Startups Companies in

Competitive Business Environment.

Procedia Manufacturing, 3, 2199-2204.

Recuperado de https://reader.elsevier.

com/reader/sd/pii/S2351978915003625?

token=1E0466A88E3D361DE249

A6292CE6CA79FBC4F85690587D02

2959AF34CDD529FA28342F6C54

5D812CAB9A9DE9DB3BA49C

\section{Newell, $\mathbf{P}$.}

2007 Biotech Firms, Biotech Politics: Negotiating GMOs in India. Journal of Environment and Development, 16(2), 183-206. Recuperado de https://journals.sagepub.com/doi/ pdf/10.1177/1070496507300920

\section{Oe, A., \& Mitsuhashi, H.}

2013

Founders' experiences for startups' fast break-even. Journal of Business Research, 66(11), 2193-2201. Recuperado de https:// www.sciencedirect.com/science/article/ abs/pii/S0148296312000306?via\%3Dihub

\section{Organización para la} Cooperación y el Desarrollo Económicos (OCDE)

Estudio multidimensional del Perú: Evaluación Inicial. Recuperado de https:// www.oecd.org/dev/MDCR\%20PERU\%20 Principales\%20mensages_FINAL.pdf

\section{Organización para la} Cooperación y el Desarrollo Económicos (OCDE)

Startup América Latina 2016 :

Construyendo un futuro innovador.

Recuperado de https://www.oecd.org/ dev/americas/Startups2016_Si-ntesis-yrecomendaciones.pdf 


\section{bibliografía}

Pastor, S.

2005 Biotecnología para conservar y utilizar sosteniblemente la agrobiodiversidad en el

Perú. En Apuntes sobre agrobiodiversidad: Conservación, biotecnología y conocimientos tradicionales (pp. 97-126). Lima: Sociedad Peruana de Derecho Ambiental.

\section{Peris, R.}

2014 Start-ups tecnológicas: el reto del crecimiento global [Tesis de licenciatura, Universidad Pontificia Comillas].

Recuperado de https://repositorio.comillas. edu/xmlui/bitstream/handle/11531/315/

TFG000135. pdf? sequence $=1$ \&isAllowed $=y$

\section{Portafolio}

8 de 'No hay nada natural en lo que comemos':

septiem-Richard Roberts, premio Nobel de

bre de Medicina, insiste en que considerar a los

2018 transgénicos como riesgosos no tiene ningún soporte científico. Grupo de Diarios América. Recuperado de https://searchproquest-com.ezproxybib.pucp.edu.pe/ docview/2101246398? accountid=28391

\section{Rank, O. N.}

2014 The effect of structural embeddedness on start-up survival: a case study in the German biotech industry. Journal of Small Business \& Entrepreneurship, 27(3), 275299. https://doi.org/10.1080/08276331.201 5.1067355

\section{Romero, G.}

2008 Biotecnología: generalidades, riesgos y beneficios. Recuperado de https:// compositae.files.wordpress.com/2015/02/ gloriaromero-1.pdf

\section{Santana, F. B., Velázquez,} J. A. D., \& Martel, M. C. V.

2006

Concepto, perspectivas y medida del crecimiento empresarial. Cuadernos de Administración, 19(31), 165-195. Recuperado de http://www.scielo.org. co/pdf/cadm/v19n31/v19n31a07.pdf? iframe $=$ true \& width $=80 \%$ \&height $=80 \%$

\section{Scott, M., \& Bruce, R.}

1987

Five stages of growth in small business. Long Range Planning, 20(3), 45-52. Recuperado de https://www. sciencedirect.com/science/article/ pii/0024630187900719?via\%3Dihub

\section{Seclen, J. P.}

2016

Gestión de la innovación empresarial: un enfoque multinivel. 360: Revista de Ciencias de la Gestión, 1(1), 16-36. Recuperado de http://revistas.pucp. edu.pe/index.php/360gestion/article/ view/14869/15410

\section{Seclen, J. P., \& Barrutia, J.}

Gestión de la Innovación Empresarial: Conceptos, Modelos y Sistemas. Lima: Fondo Editorial PUCP.

\section{Staniewski, M. W.}

2016 The contribution of business experience and knowledge to successful entrepreneurship. Journal of Business Research, 69(11), 5147-5152. Recuperado de https://www.sciencedirect.com/science/ article/abs/pii/S0148296316302582

\section{Subramaniam, M., \& Youndt,} M. A.

\section{A.}

The influence of Intellectual Capital on the

Types of Innovative Capabilities. Academy

of Management Journal, 48(3), 450-463.

https://doi.org/10.5465/AMJ.2005.17407911 


\section{bibliografía}

\section{Teece, D. J.}

2018

Business Models and dynamic capabilities.

Long Range Planning, 51(1), 40-49. https://

doi.org/10.1016/j.Irp.2017.06.007

\section{Teruel-Carrizosa, $\mathbf{M}$.}

Gibrat's law and the learning process.

Small Business Economics, 34(4), 355-373.

Recuperado de https://link.springer.com/

article/10.1007\%2Fs11187-008-9127-9

\section{Tostes, M.}

2014

Experiencias de innovación para el

desarrollo sostenible en el agro del norte

peruano: innovación, cadenas productivas y

asociatividad. Lima: Excedesa.

\section{Trigo, E., \& Villareal, F.}

La Agrobiotecnología Agrícola en las

Américas: Una mirada a la situación actual

y a las tendencias futuras. San José: IICA

Recuperado de http://repositorio.iica.int/

bitstream/11324/6785/1/BVE18039963e.pdf

Tripathi, N., Seppane, P.,

Boominathan, G., Oivo, M., \&

Liukkunen, $\mathrm{K}$.

2018

Insights into startup ecosystems through

exploration of multi-vocal literature.

Information and Software technology,

105, 56-77. https://doi.org/10.1016/j.

infsof.2018.08.005

\section{Vier, $\mathrm{H}$.}

\section{Growth of small businesses: Aliterature}

review and perspectives of studies. Gestão

\& Produção, 23(2), 419-432. Recuperado de http://www.scielo.br/scielo.php?pid=S0104-

530X2016000200419\&script=sci_

arttext\&tlng=en\#aff0100

\section{Woolfson, J.}

2013

Biotecnología, organismos genéticamente modificados y bioseguridad. En

Transgénicos: Una cuestión científica (pp. 75-78). Recuperado de https:// www.researchgate.net/profile/Cesar_ Paz-y-Mino/publication/287201502_ Transgenicos_Una_cuestion_cientifica/ links/5672d07e08aedbbb3f9f6c0e. pdf\#page $=75$

Yin, $\mathbf{R}$.

2003 Case Study Research: Design and Methods (3ra edición). Thousand Oaks, California: Sage Publications.

\section{Yin, $\mathbf{R}$.}

2018

Case Study Research: Design and Methods (6ta edición). Thousand Oaks, California: Sage Publications.

Zucker, B. L. G., Darby, M. R., \& Brewer, M. B.

1998 Intellectual Human Capital and the Birth of U.S Biotechnology Enterprises. American Economic Association, 88(1), 290-306.

Fecha de recepción: 28 de julio de 2020 Fecha de aprobación: 16 de noviembre de 2020 